\title{
A case report of Candida dubliniensis diarrhea in Systemic lupus erythematosis patient.
}

\author{
N.Girish ${ }^{1}$, K.Saileela ${ }^{2}$ \\ ${ }^{1}$ (Associate Professor, Department of Microbiology, Vydehi Institute of Medical Sciences and Research Centre, \\ Bangalore, India) \\ ${ }_{2}^{2}$ (Professor, Department of Microbiology, Kamineni Institute of Medical Sciences, Narketpally, Nalgonda \\ district, Andhra Pradesh, India)
}

\begin{abstract}
Candida dubliniensis diarrhea is an opportunistic infection in immunocompromised hosts. C.dubliniensis shares many of its phenotypic characters with the more common pathogenic yeast Candida albicans. Differentiation of the two species is important in view of the antifungal resistance reported in C.dubliniensis. A 21 year old female patient presented with diarrhea, fever, shortness of breath to the casualty. She was diagnosed with Systemic lupus erythematosis few months back when she complained of redness of face, fever, and rash on trunk and was put on corticosteroid treatment. Wet mount examination of stool revealed the presence of Candida pseudohyphae. Culture on SDA was subjected for germ tube test and chlamydospore formation, which was positive. Sparse growth on SDA at $42^{\circ} \mathrm{C}$ and no growth at $45^{\circ} \mathrm{C}$ differentiated the species from C.albicans. The patient succumbed to the infection even when the antifungal treatment was started.
\end{abstract}

Key words: Candida dubliniensis, Diarrhea.

\section{Introduction}

Candida species are frequently isolated from the stools of patients with diarrhea, primarily immunocompromised patients. Candida is also the most frequently encountered opportunistic fungal infection of the gastrointestinal tract after antibiotic exposure [1]. Candida albicans remains the most important pathogenic yeast. Since 1995, a novel Candida species has been identified and named Candida dubliniensis. This species shares many phenotypic characteristics with Candida albicans, including the ability to produce germ tubes and chlamydospores. The identification of this species in routine cultures is essential in order to further understand the epidemiology, to better define the role of Candida dubliniensis as a potential pathogen and its susceptibility to develop resistance to the antifungal agents [2].

\section{Case report}

A 21 year old female patient presented with diarrhea and fever since 10 days, cough and shortness of breath since 7 days to the casualty department of Kamineni Institute of Medical Sciences, Narketpally, Nalgonda district, Andhra Pradesh. The patient was diagnosed with Systemic lupus erythematosis 2 months back when she presented with redness of face, fever and rash on trunk and extensor aspect of upper limb and was put on corticosteroids treatment.

On examination hyper pigmented macular papular scaly lesions were seen over sun exposed areas. Her temperature was $102^{\circ} \mathrm{C}$, pulse $120 / \mathrm{min}$, BP $110 / 70$, and Respiratory rate $42 / \mathrm{min}$.

Laboratory tests revealed $\mathrm{Hb}-8.8 \%$, RBC- 3.2million/ml, total count- 2000/ml, Neutrophils-50\%, Lymphocytes-40\% Eosinophils-2\% and Monocytes-8\%, Platelets-80000/ml. Peripheral smear revealed normocytic normochromic anaemia and Malarial parasite negative. RBS is within normal limits. Triglycerides and LDH elevated. HIV and HBsAg negative. Stool wet mount revealed the presence of Candida pseudohyphae (Figure 1). The Gram stain of the stool smear revealed Gram positive oval budding yeast like cells (Figure 2). ANA was positive and anti ds DNA negative. Chest $\mathrm{X}$ ray revealed bilateral pulmonary infiltrates.

The patient's stool sample was inoculated onto SDA with Gentamicin and after overnight incubation significant number of small cream colored colonies was seen. The germ tube test was positive (Figure 3). Growth on cornmeal agar revealed chlamydospore formation (Figure 4). The species was identified as C.dubliniensis based on sparse growth on SDA at $42^{\circ} \mathrm{C}$ and no growth at $45^{\circ} \mathrm{C}$ and positive urease test.

The patient was treated with Oxygen inhalation, IV fluids, Asthalin nebulisation, Inj. Ciprofloxacin, Metronidazole, Cap Doxycyline, Tab Ranitidine, Sporolac, Tab Prednisolone $40 \mathrm{mg}$ and antifungal drugs was started after getting stool culture report. Unfortunately the patient succumbed to her illness.

\section{Discussion}

Candida dubliniensis is a relatively newly described species of the most protean genus of pathogenic yeasts. The vast majority of C.dubliniensis isolates identified 
to date have been recovered from the oral cavities HIV infected individuals, particularly those suffering from recurrent episodes of oral candidiasis [3]. However a small number of isolates associated with other anatomical sites, including the vagina and the lung, have been reported [4].

Here we are reporting a fatal case of diarrhea caused by C.dubliniensis in a patient suffering from an immunocompromised condition SLE. As this species shares many phenotypic characters with C.albicans differentiation is very important in view of fluconazole resistance associated with this species. While the use of a number of phenotypic characters e.g., chlamydospore formation, carbohydrate assimilation, colonial coloration on differential media such as CHROM agar Candida, methyl blue Sabouraud agar, has been investigated, none have provided a completely reliable method for the differentiation of the two species [5].

The most reliable methods include PCR, DNA finger printing with repetitive sequence containing DNA probes and Pulsed field gel electrophoresis [6]. Although they are very effective, these techniques cannot be conducted for routine diagnostic purposes in most standard microbiological laboratories. It has previously been suggested that the two species could be distinguished by the inhibition of the growth of C.dubliniensis at $42^{\circ} \mathrm{C}$. However, some studies have shown that approximately $10 \%$ of C.dubliniensis isolates were able to grow, albeit poorly, at this elevated temperature. Studies have shown that complete inhibition of growth of C.dubliniensis at $45^{\circ} \mathrm{C}$ would provide a simple, inexpensive and reliable means of differentiation of the two species [7].

\section{Conclusion}

C.dubliniensis is an opportunistic infection. Accurate identification of C.dubliniensis is essential especially in immunocompromised patients in view of antifungal resistance reported in this species.

\section{References}

[1] Vaishnavi C, Kaur S, Prakash S. Speciation of Fecal Candida isolates in antibiotic associated diarrhea in non-HIV patients. Jpn.J.Infect. Dis., 61, 1-4, 2008.

[2] Anane S, Kallel K, Kaouech E, Belhaj S, Chaker E. Candida dubliniensis: a novel emerging species. Ann Biol Clin (Paris). 2007 JanFeb; 65(1): 13-9.

[3] Coleman DC, Sullivan DJ, Bennett DE, Moran GP, Barry H J, and Shanley DB. $1997 . \quad$ Candidiasis: the emergence of a novel species, Candida dubliniensis. AIDS 11:557-567.

[4] Sullivan D, Coleman D. 1998. Candida dubliniensis: characteristics and identification. J. Clin. Microbiol. 36:329-334

[5] Schoofs A, Odds FC, Colebunders R, Ieven M, and Goosens H. 1997. Use of specialised isolation media for recognition and identification of Candida dubliniensis isolates from HIV-infected patients. Eur. J. Clin. Infect. Dis. 16:296-300.

[6] Khlif M, Sellami A, Sellami H, Makni F, Ayadi A. Candida dubliniensis: Identification methods and epidemiologic implication. Pathol Biol (Paris). 2011 Jun; 59(3):166-72.

[7] Pinjon E,Sullivan D, Salkin I,Shanely D,Coleman D. Simple, inexpensive and reliable method for differentiation of Candida dubliniensis from Candida albicans. J Clin Microbiol, July 1998, vol 36, no7, p 2093-2095.

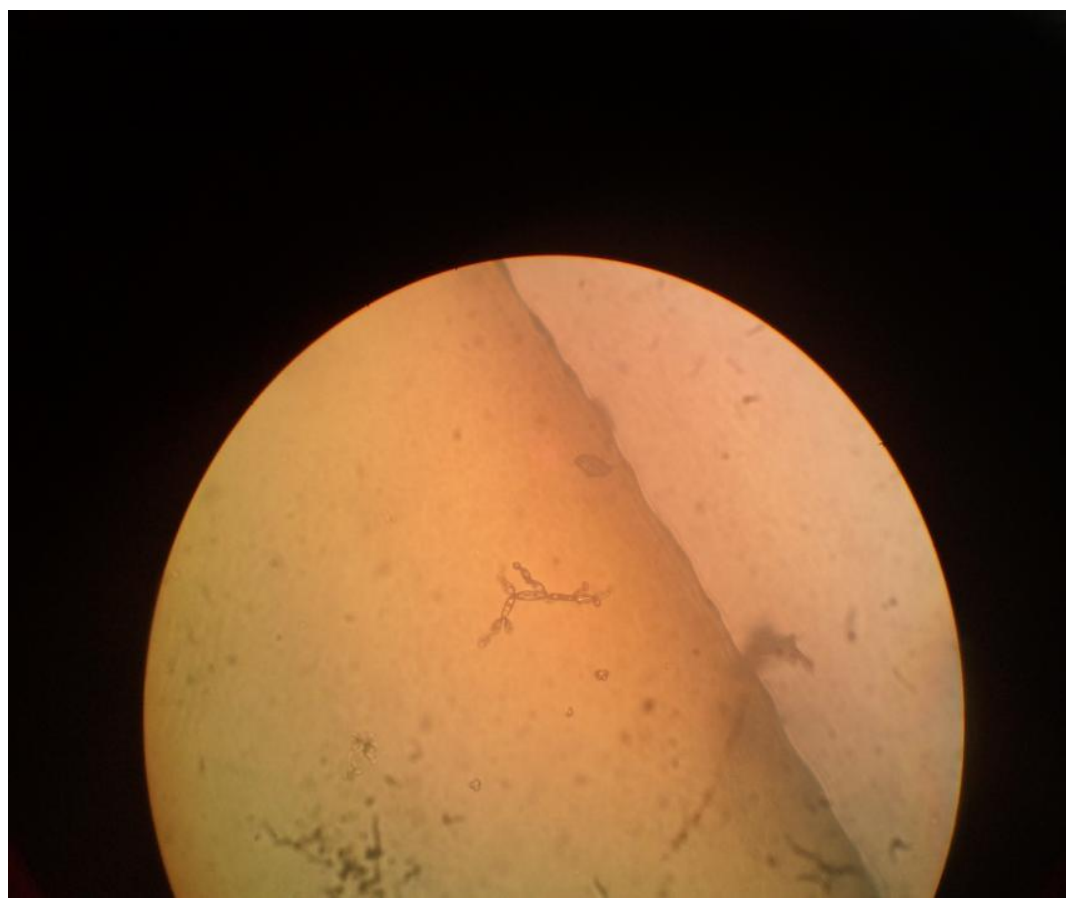

Figure-1: Wet mount preparation of the stool sample revealing Candida pseudohyphae. 


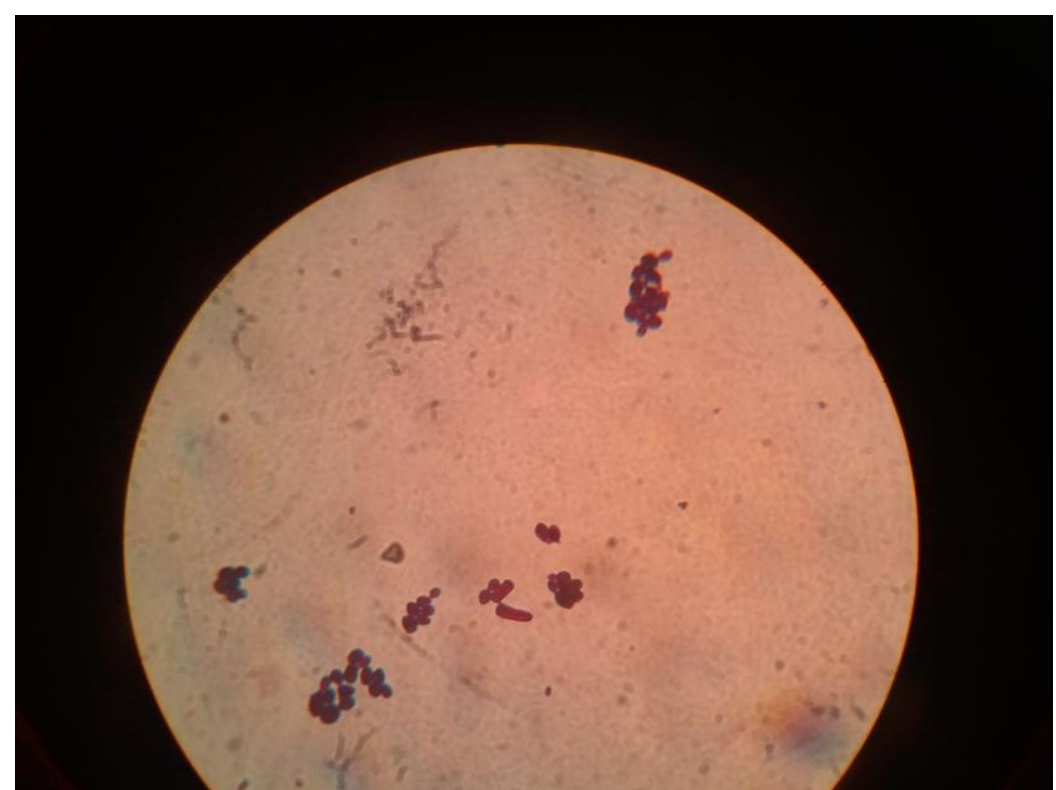

Figure-2: Gram stain of the stool smear showing gram positive budding yeast like cells.

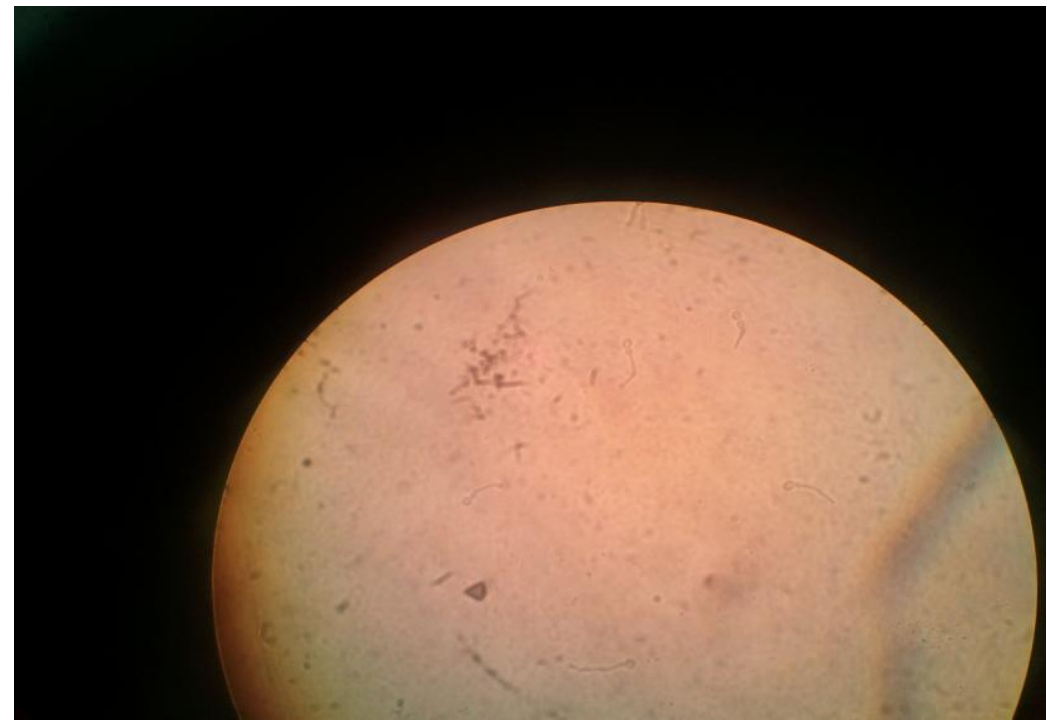

Figure-3: Germ tube test.

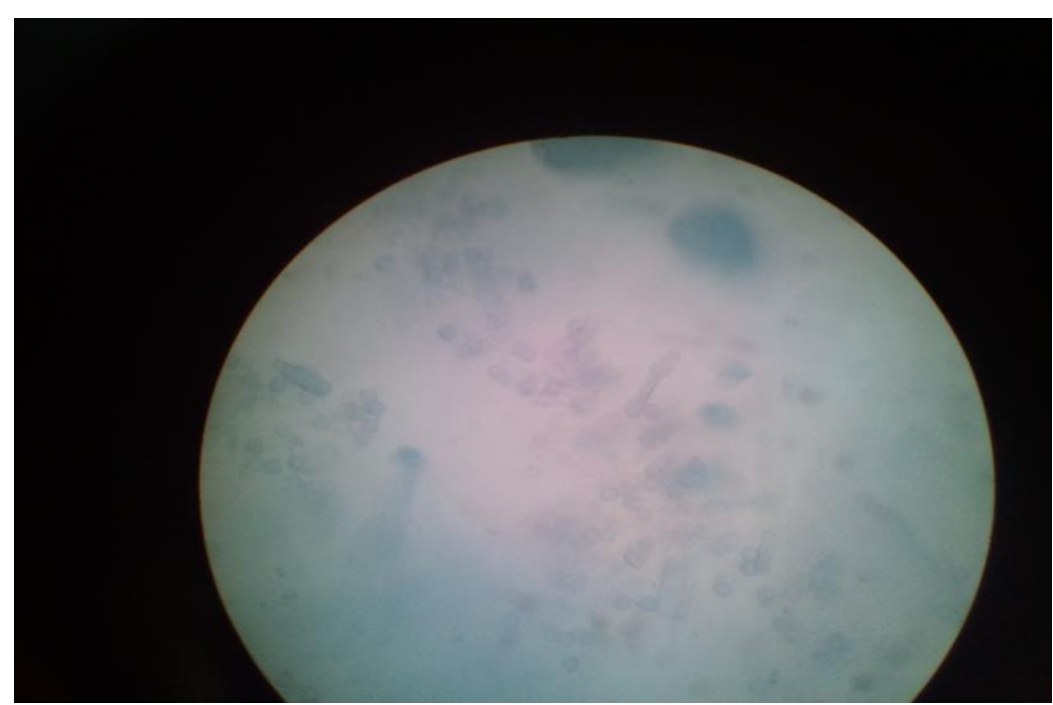

Figure-4: Chlamydospore formation. 